Roy P.C. Kessels ${ }^{1,2, *}$

Pieter Spee ${ }^{1}$ Angelique W. Hendriks

'Radboud University Nijmegen, Donders Institute for Brain,

Cognition and Behaviour,

P.O. Box 9104, 6500 HE Nijmegen,

The Netherlands

${ }^{2}$ Radboud University Nijmegen Medical

Centre, Department of Medical Psychology,

P.O. Box 9101, 6500 HB Nijmegen,

The Netherlands

${ }^{3}$ Radboud University Nijmegen,

Behavioural Science Institute, Department

of Special Education

P.O. Box 9104,6500 HE Nijmegen,

The Netherlands

Received 31 August 2010 accepted 16 September 2010

\section{Introduction}

There is abundant evidence that individuals diagnosed with autism spectrum disorders (ASD) have difficulty attributing mental states, such as intentions, desires and thoughts to oneself and others [1]. Consequently, they show difficulties in social interaction and communication [2]. Also, several studies have shown that, relative to controls, children with autism look less frequently and in an atypical manner at faces [3]. For example, they show less fixation on salient facial features such as the eyes or mouth than controls and more fixation on facial regions without core features [4]. Autism has also been associated with facial perception deficits, such as memory for faces [5] or incidental learning of faces [6]. However, with respect to the perception of facial emotion, results are mixed. Whereas several studies found autistic individuals to be impaired in recognizing facial expressions [7], others have failed to find such a deficit [8]. Possibly, more

\title{
PERCEPTION OF DYNAMIC FACIAL EMOTIONAL EXPRESSIONS IN ADOLESCENTS WITH AUTISM SPECTRUM DISORDERS (ASD)
}

\section{Abstract}

Previous studies have shown deficits in the perception of static emotional facial expressions in individuals with autism spectrum disorders (ASD), but results are inconclusive. Possibly, using dynamic facial stimuli expressing emotions at different levels of intensities may produce more robust results, since these resemble the expression of emotions in daily life to a greater extent. 30 Young adolescents with high-functioning ASD (IQ>85) and 30 age- and intelligence-matched controls (ages between 12 and 15) performed the Emotion Recognition Task, in which morphs were presented on a computer screen, depicting facial expressions of the six basic emotions (happiness, disgust, fear, anger, surprise and sadness) at nine levels of emotional intensity (20-100\%). The results showed no overall group difference on the ERT, apart from a slightly worse performance on the perception of the emotions fear $(p<0.03)$ and disgust $(p<0.05)$. No interaction was found between intensity level of the emotions and group. Highfunctioning individuals with ASD perform similar to matched controls on the perception of dynamic facial emotional expressions, even at low intensities of emotional expression. These findings are in agreement with other recent studies showing that emotion perception deficits in high-functioning ASD may be less pronounced than previously thought.

Keywords

Autism spectrum disorders $\cdot$ PDD-NOS $\cdot$ Asperger $\cdot$ Social cognition $•$ Emotion perception

(c) Versita Sp. z o.o.

general visuoperceptual difficulties underlie these deficits [9]. Recently, it was suggested that the face processing deficit in autism may be caused by delayed emergence of the global properties of facial stimuli, resulting in piecemeal processing of faces (A.W. Hendriks and P. Benson, submitted for publication). As face processing is best when both local and global information is used [10], this may lead to diminished performance, especially when face perception is relatively difficult such as is the case with complex emotions [11]. The visual pathway sub-serving the processing of global form is the magnocellular pathway [12], which as evidence suggests is indeed functioning abnormally in autism [13]. Another major function of the magnocellular pathway is the processing of movement [14], which is also compromised in autism $[14,15]$. The question, therefore, arises whether the use of moving facial expressions is perhaps a more sensitive means of detecting deficits in autism in processing facial expressions, especially if these expressions would be displayed at different intensities [16]. Apart from the expected greater sensitivity, the use of such stimuli would also have the advantage of having a higher ecological validity than the static photographs normally used in studies of face perception in autism. The current study examined emotional face perception in young adolescents with autism spectrum disorders using dynamic stimuli (i.e., morphs in which a neutral face gradually changes into an emotional expression), taking into account different levels of emotional intensities.

\section{Experimental Procedures}

\subsection{Participants}

The individuals with autism spectrum disorders were 30 pupils ( 27 males) of "De Isselborgh" in Doetinchem, the Netherlands, a special education institute. All children fulfilled the criteria for autism spectrum

*E-mail:r.kessels@donders.ru.nl 
disorders (ASD) according to the DSM-IV-TR (APA, 1994) and had normal intelligence levels (IQ>85). Twenty-two children were diagnosed with Pervasive Developmental Disorder Not Otherwise Specified (PDD-NOS), 7 with Autistic Disorder and 1 with Asperger's Disorder. The study was approved by the Institutional Review Board of the institute and informed consents were obtained from the children and their parents (or legal guardians), in accordance with the declaration of Helsinki. Controls were 30 children (27 males) from secondary schools in Oss ("Maaslandcollege") and Ravenstein ("Hooghuis Lyceum"), the Netherlands. Intelligence levels (deviation IQ) were determined using Dutch versions of established intelligence tests, i.e. Raven's Progressive Matrices and Wechsler's Intelligence Scale for Children (WISC-R/ WISC-III), or validated Dutch intelligence test batteries for use in educational settings, that is, the Groningen Final Examination Primary Education (GALO) and the Groningen/ Netherlands Educational Intelligence Test (GIVO/NIO). None of the control participants had a history of psychiatric or neurological disease. All participants had normal or corrected-to-normal vision. The two groups did not differ with respect to age (mean patients 13.3, SD=1.0; mean controls 13.1,
$\mathrm{SD}=1.0 ; \mathrm{t} 58=0.51$ ), IQ (mean patients 104.6, $\mathrm{SD}=7.8$; mean controls 107.1, $\mathrm{SD}=12.1$; $\mathrm{t} 51=0.40$ ) or sex distribution (Mann-Whitney $\mathrm{U}=450.0$ ).

\subsection{Materials and procedure}

The Emotion Recognition Task (ERT) $[16,17]$ was administered to all participants. In this task, short video clips were presented, showing a neutral face gradually changing into one of the six basic emotions (happiness, anger, disgust, fear, sadness and surprise). Four actors (2 men) mimicked these facial expressions and their expressions were subsequently rated by a panel. Different computer-generated intensity levels were constructed for each emotion to enable real-time interactive morphing between two end points (the first being always neutral, see Figure 1 for an overview). Intensities increased gradually from $0-20 \%$ in the first trials, $0-30 \%$ in the subsequent trials, and so on with steps of $10 \%$ increase, until $0-100 \%$ (fullblown emotion). After each trial, participants had to indicate which type of emotion was shown from six verbal emotion labels that were presented at the left side of the screen with no time restrictions. The video clips were presented in nine blocks of 24 trials. The task was presented using a Compaq Pressario C500 personal computer with a 15 inch computer monitor (resolution $1280 \times 800$, refresh rate $60 \mathrm{~Hz}$ ). Viewing distance was approximately $80 \mathrm{~cm}$. All participants were examined individually in a quiet, well-lit room and were instructed by the examiner. After having performed three practice trials, participants were prompted to ask questions, after which the actual test commenced.

\section{Results}

Figure 2 shows the results for the controls and the ASD group for all emotion types and different intensity levels. General linear model (GLM) analysis with Emotion (happiness, anger, disgust, fear, sadness and surprise) and Intensity (9 levels) as within-subject factors and Group (ASD, control) as between-subject factor showed overall main effects for Emotion (F5,54=347.4, $\quad \mathrm{p}<0.0005)$ and Intensity $(F 8,51=48.6, p<0.0005)$, in accordance with previous findings [17]. No overall Group effect was found $(F 1,58=0.8)$. In addition, an interaction between Emotion and Intensity was found $(F 40,19=5.0, p<0.0005)$, as well as a significant interaction between Emotion type and Group ( $F 5,54=2.3, p=0.05)$. All other interactions were not significant (all F-values $<0.95$ ). Subsequent $9 \times 2$ GLM analyses for

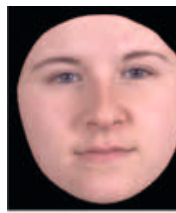

$0 \%$
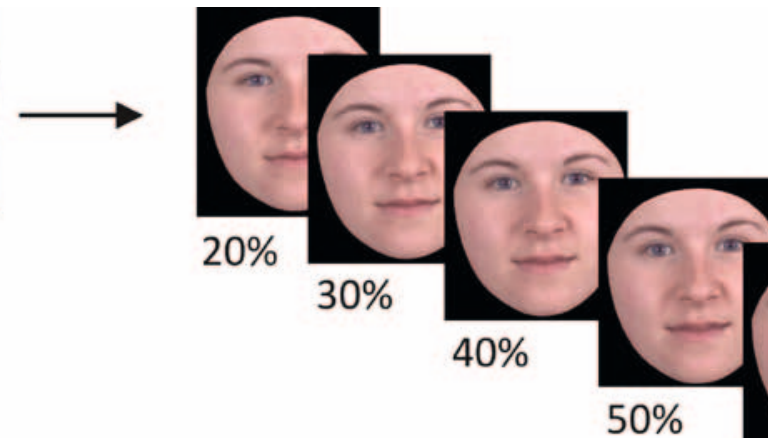

Figure 1. Example of the different levels of emotional intensity showing the nine morphing steps from neutral (0\%) to a $100 \%$ surprised face. 

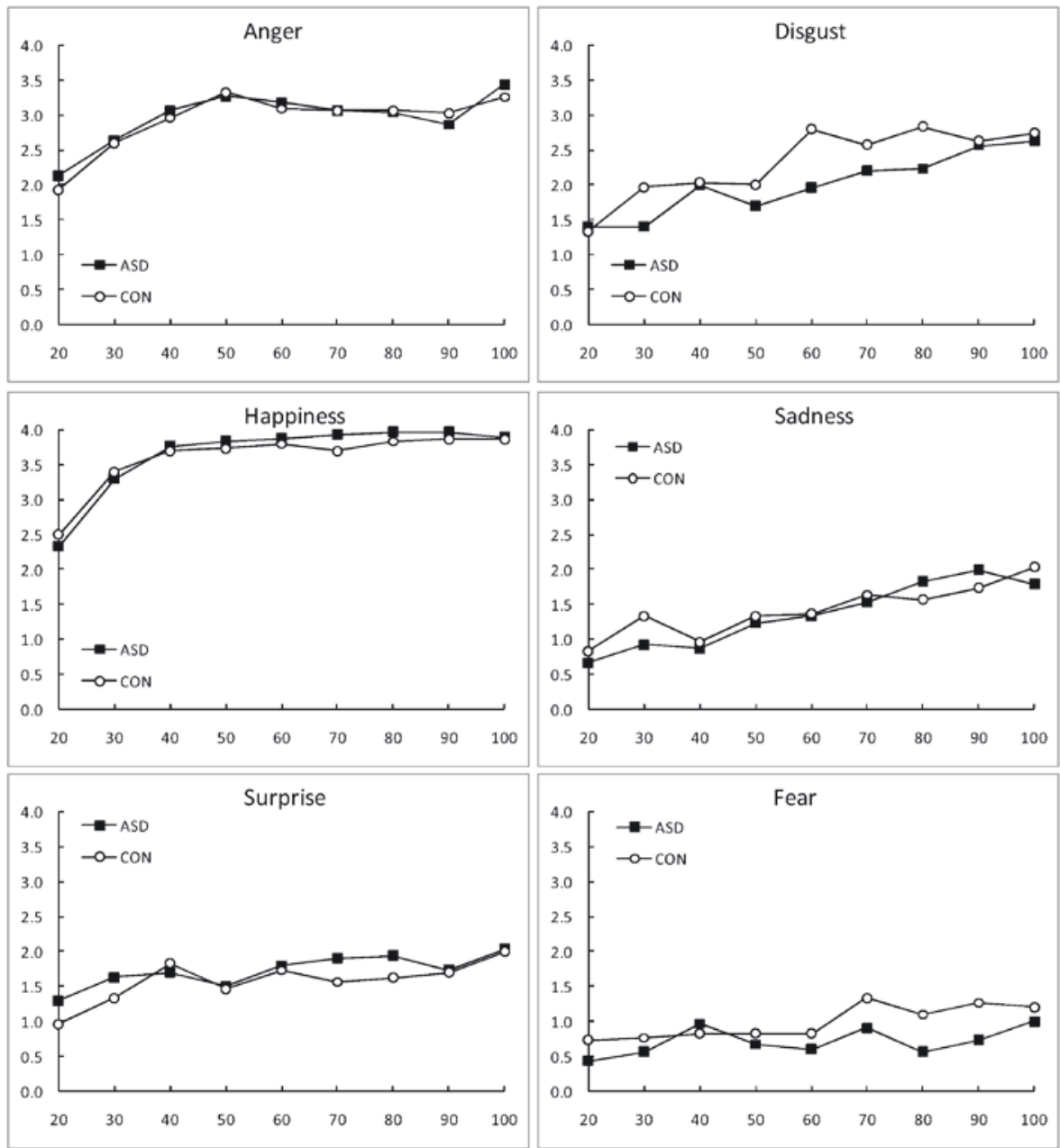

Figure 2. Mean performance ( $y$-axis; $\max =4$ ) for the 6 basic emotions presented at different intensity levels ( $x$-axis; 20-100\%) for the young adolescents with Autism Spectrum Disorders (ASD) and the matched controls.

the separate emotions demonstrated main effects of Intensity for all Emotion types. The largest effects of Intensity were found for the emotions Happiness ( $\mathrm{F} 8,51=34.4, \mathrm{p}<0.0005)$, Sadness $(F 8,51=12.7, p<0.0005)$ and Anger $(F 8,51=12.7, p<0.0005)$. The smallest effects of Intensity were observed for the emotions Disgust $\quad(F 8,51=8.7, \quad p<0.0005)$, Surprise $(\mathrm{F} 8,51=4.8, \mathrm{p}<0.0005)$, and Fear $(\mathrm{F} 8,51=2.6$, $\mathrm{p}<0.02$ ). In addition, a main effect of group was found for the emotions Fear $(F 1,58=3.8$, $\mathrm{p}<0.03$ one-tailed) and Disgust $(F 1,58=3.1$, $p<0.05$ one-tailed). None of the Group $\times$ Intensity interactions was significant (all F-values<1.6).

\section{Discussion}

The present study set out to examine the perception of dynamic facial emotional expressions at different levels of intensity in young adolescents with ASD and age- and IQ-matched controls. Overall, the ASD group performed equivalent to the controls on the emotion perception task, with the exception of a slightly worse performance of the ASD group on the emotions fear and disgust. In addition, although a general effect of emotion intensity was found (i.e., emotional expressions were recognized worse at lower than at higher intensities), there was no interaction between emotional intensity and group. Thus, the ASD group did not perform differentially worse on the trials with less intense emotional expressions compared to the controls.

Although several other studies have also failed to find evidence for an emotional face processing deficit in autism, we argued that this could be due to their use of static full-blown emotional stimuli that may not be very sensitive to subtle deficits in emotion perception. Instead, the present study used dynamic displays of emotional expressions, presenting them at intensities of even very low levels. Both movement and low intensities were expected to reveal 
a face processing deficit in our participant group with ASD even if subtle, in line with recent evidence suggesting a magnocellular deficit in autism that would hamper the processing of motion and global form [1315]. Our results, however, did not reveal deficits in the perception of dynamic facial expressions in high-functioning young adolescents with ASD. Possibly, since deficits have been demonstrated in children with ASD more consistently, in our sample of high-functioning young adolescents compensatory strategies may have helped to overcome underlying deficits in low-level perception.

Compensatory mechanisms may have also played a role in a recent study [18] that used a more ecologically valid approach in taking the context of emotional expressions into account. Here, the use of visual context (i.e., depicting a scene in which one of the persons visible expresses an emotion) did neither result in group differences between the ASD and control group on the perception of static isolated faces, nor on the identification of emotional expressions presented in a visual context, leading the authors to conclude that deficits in emotion perception in high-functioning ASD may be less pronounced than previously thought.

Although our findings appear to support the latter conclusion, we cannot conclude that the processing of emotional stimuli is unaffected in all patients with ASD. That is, our sample was limited to young adolescents with high-functioning ASD. Possibly, deficits in emotion perception may be more marked in more classic autism. However, findings in lowfunctioning autism may be more difficult to interpret because lower levels of intelligence may also affect performance on emotion perception task. There is indeed evidence that large group differences on emotion perception tasks disappeared after controlling for (verbal) intelligence levels $[8,18]$. In turn, it is possible that high-functioning ASD individuals are able to compensate for neurocognitive deficits in emotion processing, resulting in an apparently normal performance on experimental paradigms. A recent $\mathrm{AMRI}$ study [19] has examined neural activation related to the perception of emotional morphs, similar to our morphs showing a neutral face changing into a full emotional expression, compared to static emotion expressions in ASD. In this study, ASD individual demonstrated altered brain activation in the social brain, notably the amygdala, superior temporal sulcus and the fusiform gyrus, although at a behavioral level, no differences are reported between the ASD group and the matched controls.

In all, ours is the first study to examine the perception of morphed emotional facial expressions at different levels of intensity in high-functioning young adolescents with ASD. The results did not show marked performance differences between ASD individuals and matched controls, not even on lower intensity levels that may be more sensitive to subtle deficits. These findings are in agreement with other studies indicating that neither the perception of static facial expressions in isolation, nor facial expressions supported by emotional wholebody movements, nor expressions presented in a visual context are grossly impaired in high-functioning ASD. Future studies should examine whether the perception of dynamic facial emotional expressions is impaired in either children with ASD or in people with low-functioning autism.

References

[1] Yirmiya N., Erel O., Shaked M., Solomonica-Levi D., Meta-analyses comparing Theories-of-Mind abilities of individuals with autism, individuals with mental retardation, and normally developing individuals, Psychol Bull, 1998, 124, 283-307

[2] Rutter M., Autism: its recognition, early diagnosis, and service implications, J Dev Behav Pediatr, 2006, 27 Suppl 2, S54-58

[3] Van der Geest J.N., Kemner C., Verbaten M.N., van Engeland H., Gaze behaviour of children with pervasive developmental disorder toward human face, J Child Psychol Psychiatry, 2002, 43, 669-667

[4] Pelphrey K.A., Sasson N.J., Reznick J.S., Paul G., Goldman B.D., Piven J., Visual scanning of faces in autism, J Autism Dev Disord, 2002, 32, 249-261

[5] Williams D.L., Goldstein G., Minshew N.J., Impaired memory for faces and social scenes in autism: clinical implications of the memory disorder, Arch Clin Neuropsychol, 2005, 20, 1-15

[6] De Gelder B., Vroomen J., van der Heide L., Face recognition and lipreading in autism, Eur J Cogn Psychol, 1991, 3, 69-86

[7] Kuusikko S., Haapsamo H., Jansson-Verkasalo E., Hurtig T., Mattila M.L., Ebeling $\mathrm{H}_{\text {., }}$ et al., Emotion recognition in children and adolescents with autism spectrum disorders, J Autism Dev Disord, 2009, 39, 938-945
[8] Ozonoff S., Pennington B.F., Rogers S.J. Are there specific emotion perception deficits in young autistic children?, J Child Psychol Psychiatry, 1990, 31, 343-361

[9] Baruth J.M., Casanova M.F., Sears L., Sokhadze E., Early-stage visual processing abnormalities in high-functioning autism spectrum disorder (ASD), Transl Neurosci, 2010, 1, 177-187

[10] Halit H., de Haan M., Schyns P.G., Johnson M.H., Is high-spatial frequency information used in the early stages of face detection?, Brain Res, 2006, 1117, 154-161

[11] Golan O., Baron-Cohen S., Hill J, The Cambridge Mindreading (CAM) Face-Voice Battery: testing complex emotion recognition in adults with and without Asperger syndrome, J Autism Dev Disord, 2006, 36, 169-183

[12] Milner A.D., Goodale M.A., The visual brain in action. Oxford University Press, Oxford, 1995

[13] McCleery J.P., Allman E., Carver L., Dobkins K.R., Abnormal magnocellular pathway visual processing in infants at risk for autism, Biol Psychiatry, 2007, 62, 1007-1014

[14] Spencer J., O'Brien J., Riggs K., Braddick O., Atkinson J., Wattam-Bell J., Motion processing in autism: evidence for a dorsal stream deficiency, Neuroreport, 2000, 11, 2765-2767 
[15] Milne E., Swettenham J., Hansen P., Campbell R., Jeffries H., Plaisted K., High motion coherence thresholds in children with autism, J Child Psychol Psychiatry, 2002, 43, 255-263

[16] Montagne B., de Geus F., Kessels R.P.C., Denys D., De Haan E.H.F., Westenberg H.G.M., Perception of facial expressions in obsessivecompulsive disorder: a dimensional approach, Eur Psychiatry, 2008, 23, 26-28

[17] Montagne B., Kessels R.P.C., De Haan E.H.F., Perrett D.I., The Emotion Recognition Task: a paradigm to measure the perception of facial emotional expressions at different intensities, Percept Mot Skills, 2007, 104, 589-598

[18] Wright B., Clarke N., Jordan J., Young A.W., Clarke P., Miles J., et al., Emotion recognition in faces and the use of visual context in young people with high-functioning autism spectrum disorders, Autism, 2008, 12, 607-626

[19] Pelphrey K.A., Morris J.P., McCarthy G., Labar K.S., Perception of dynamic changes in facial affect and identity in autism, Soc Cogn Affect Neurosci, 2007, 2, 140-149 